\title{
Sample Concentration and Processing
}

\author{
Lane C. Sander \\ National Institute of Standards and Technology, \\ Gaithersburg, MD 20899, USA \\ lane.sander@nist.gov
}

Video DOI: http://doi.org/10.18434/T4DK5T

Key words: concentration; evaporation; method development; quantitation; sample processing; solvent exchange.

Accepted: December 1, 2016

Published: January 12, 2017

https://doi.org/10.6028/jres.122.011

\section{Summary}

Sample concentration is a common step in sample preparation prior to analysis that is performed to increase method sensitivity or to exchange solvent environment. Sample concentration can be thought of as a separation technique, since the more volatile component of a mixture is preferentially removed from less volatile components. In most, but not all cases, the more volatile components are not recovered. This presentation will provide an overview of different approaches used to concentrate samples, with brief demonstrations of the instruments used, including a needle evaporator, automated evaporator, rotary evaporator, and SpeedVac concentrator. An example will also be included of an instrument that is primarily intended for sample processing reactions (HotBlock digestor). ${ }^{1}$

\footnotetext{
${ }^{1}$ Contribution of the National Institute of Standards and Technology. Not subject to copyright. Certain commercial equipment, instruments, or materials are identified to specify adequately the experimental procedure. Such identification does not imply recommendation or endorsement by the National Institute of Standards and Technology, nor does it imply that the materials or equipment identified are the best available for the purpose.
} 